\title{
Synthesis and Characterization of New Chiral Monoanionic [ON] Ancillary Phenolate Ligands
}

\author{
Pascal Binda1,2*, Leslie Glover ${ }^{2}$ \\ ${ }^{1}$ Department of Chemistry and Forensic Science, Savannah State University, \\ Savannah, GA, USA \\ ${ }^{2}$ Science Division, Southern Wesleyan University, Central, SC, USA \\ Email: bindap@savannahstate.edu
}

Received 25 June 2014; revised 13 August 2014; accepted 28 August 2014

Copyright (C) 2014 by authors and Scientific Research Publishing Inc.

This work is licensed under the Creative Commons Attribution International License (CC BY). http://creativecommons.org/licenses/by/4.0/

c) (i) Open Access

\begin{abstract}
Three new chiral monoanionic [ON] ancillary phenolate ligands with varying pendant arms have been synthesized in moderate to high yields (50\% - 85\%) via Mannich-type condensation reaction of chiral substituted phenol, formaldehyde and (+)-bis-[(R)-1-phenylethyl]amine. These new organic compounds were fully characterized via nuclear magnetic resonance spectroscopy $\left({ }^{1} \mathrm{H}\right.$ and ${ }^{13} \mathrm{C}$ ) and elemental analysis. The newly synthesized ligands are suitable candidates for metalcatalyzed ring-opening of lactones and asymmetric catalysis.
\end{abstract}

\section{Keywords}

Phenolate Ligands, Mannich Condensation Reaction, Ring-Opening Polymerization of Lactones

\section{Introduction}

Ligand design has been of great importance in asymmetric catalysis and ring opening polymerization of lactones due to the fact that the reactivity and selectivity of metal catalysts are largely determined by the ancillary ligands [1]-[7]. One of the challenges is to derive efficient chiral catalysts for asymmetric induction in different substrates with subtle variations. Since it is not expected that a single catalyst will work for a wide range of substrates, an efficient strategy towards new catalysts would be the design of a search pathway that provides access to a large number of structurally similar ligands with tunable yet diverse substituents [8]. Indeed, many researchers have sought to develop asymmetric catalysts by screening a large pool of chiral ligands [9]-[12].

${ }^{*}$ Corresponding author.

How to cite this paper: Binda, P. and Glover, L. (2014) Synthesis and Characterization of New Chiral Monoanionic [ON] Ancillary Phenolate Ligands. International Journal of Organic Chemistry, 4, 182-188. 
Aminophenolate ligands have received great attention in metal-catalyzed ring-opening polymerization (ROP) of lactones due to the potential to fine tune the steric and electronic properties by varying the substituent groups and pendant side-arms, as well as their inexpensive synthetic strategies [13]-[22]. In fact, well-defined metal complexes of phenolate as ancillary ligands have been studied intensively to investigate the electronic and steric properties of the central metal and their effects in ROP of cyclic esters [13]-[46]. However, given their widespread application, it is somewhat surprising that the chiral variants of aminophenolate ligands are relatively lacking in the literature. The introduction of an aromatic ring in the pendant side arm, the resonance in the backbone is attenuated in comparison with the regular ligands and this may offer some unique opportunities for electronic differentiation and stereocontrol upon coordination [47]. Of particular interest are ligands with chiral pendant substituents that are in close proximity with the open coordination site at the periphery where catalysis occurs. Discussed herein is the synthesis of new chiral [ON] aminophenolate ligands as potential ancillary ligands in asymmetric catalysis and ring-opening polymerization of lactones.

\section{Experimental}

\subsection{General}

Deuterated solvents were purchased from Cambridge Isotope Laboratory and used as received. 2,4-di-tertbutylphenol, 2,4-dimethylphenol, 2,4-di-tert-pentylphenol, $37 \mathrm{wt} \%$ formaldehyde, and N-methylbenzylamine were purchased from Acros Organic and used as received. 2-tert-butyl-4-methylphenol and (+)-bis-[(R)-1-phenylethyl] amine were purchased from Aldrich while 4-tert-butyl-2-methylphenol was purchased from Fluka and used as received. All ${ }^{1} \mathrm{H}$ and ${ }^{13} \mathrm{C}$ NMR spectra were recorded on a JEOL ECX-300 MHz NMR spectrometer and referenced to $\mathrm{CDCl}_{3}$. Elemental analyses were performed by Midwest Microlab, Indianapolis, IN. Melting points were obtained on a Mel-Temp apparatus and are uncorrected.

\subsection{Synthesis of Ligands}

\subsubsection{HL HL $^{\mathrm{a}}$}

2,4-Di-tert-butylphenol (1.834 g, $8.87 \mathrm{mmol}), 37 \mathrm{wt} \%$ formaldehyde (0.266 g, $8.87 \mathrm{mmol})$, and (+)-bis-[(R)-1phenylethyl] amine $(2.000 \mathrm{~g}, 8.87 \mathrm{mmol})$ were dissolved in ethanol $(13 \mathrm{~mL})$. The resulting solution was heated at reflux for $18 \mathrm{~h}$ and then cooled to room temperature. Solvent and water were removed using high vacuum Schlenk line to obtain pale yellow oily compound, which was purified by column chromatography (5\% ethyl acetate and 95\% hexane). (2.198 g, 55.9\%). Elemental analysis: (Found: $\mathrm{C} 83.22, \mathrm{H}$ 9.03, N 3.38. $\mathrm{C}_{31} \mathrm{H}_{41} \mathrm{NO}$ requires C 83.922, H 9.315, N 3.157. ${ }^{1} \mathrm{H}$ NMR (300 MHz; $\mathrm{CDCl}_{3}$; $\left.298 \mathrm{~K}\right) 1.30$ (s, 9H, Ar ${ }^{\mathrm{t}} \mathrm{Bu}$ ), 1.37 (d, 3H, $J=$ $6.87 \mathrm{~Hz}, \operatorname{ArCH}(M e) \mathrm{N}), 1.44\left(\mathrm{~s}, 9 \mathrm{H}, \mathrm{Ar}^{\mathrm{t}} \mathrm{Bu}\right), 1.52(\mathrm{~d}, 3 \mathrm{H}, J=6.87 \mathrm{~Hz}, \operatorname{ArCH}(M e) \mathrm{N}), 3.71(\mathrm{~d}, 1 \mathrm{H}, J=14.76 \mathrm{~Hz}$, $\left.\operatorname{ArCH}_{2} \mathrm{~N}\right), 4.12(\mathrm{q}, 1 \mathrm{H}, J=6.87 \mathrm{~Hz}, \operatorname{ArCH}(\mathrm{Me}) \mathrm{N}), 4.29\left(\mathrm{~d}, 1 \mathrm{H}, J=14.76 \mathrm{~Hz}, \operatorname{ArCH}_{2} \mathrm{~N}\right), 4.84(\mathrm{br}, 1 \mathrm{H}$, $\operatorname{ArCH}(\mathrm{Me}) \mathrm{N}), 6.64(\mathrm{~d}, 1 \mathrm{H}, J=8.22 \mathrm{~Hz}, \operatorname{ArH}), 7.12(\mathrm{~d}, 1 \mathrm{H}, J=8.22 \mathrm{~Hz}, \mathrm{ArH}), 7.30$ - 7.40 (br, 10H, ArH), 11.12 (s, $1 \mathrm{H}, \mathrm{ArOH}) .{ }^{13} \mathrm{C}\{\mathrm{H}\}$ NMR $\left(75 \mathrm{MHz} ; \mathrm{CDCl}_{3} ; 298 \mathrm{~K}\right) 24.9(\mathrm{ArCH}(\mathrm{Me}) \mathrm{N}), 29.7\left(\mathrm{ArCMe}_{3}\right), 31.8\left(\mathrm{ArCMe}_{3}\right)$, $34.7\left(\mathrm{ArCMe}_{3}\right), 34.9\left(\mathrm{ArCMe}_{3}\right), 51.3(\mathrm{ArCH}(\mathrm{Me}) \mathrm{N}), 53.3\left(\mathrm{ArCH}_{2} \mathrm{~N}\right), 116.0,123.6,126.9,127.1,128.3,128.5$, 128.6, 142.9, 152.1 (all $\mathrm{ArC}$ ).

\subsubsection{HL}

2-Tert-butyl-4-methylphenol (2.189 g, $13.31 \mathrm{mmol}), 37 \mathrm{wt} \%$ formaldehyde (0.400 g, $13.31 \mathrm{mmol})$, and (+)-bis[(R)-1-phenylethyl] amine (3.000 g, $13.31 \mathrm{mmol})$ were dissolved in ethanol $(13 \mathrm{~mL})$. The resulting solution was heated at reflux for $18 \mathrm{~h}$ and then cooled to room temperature. Solvent and water were removed using high vacuum Schlenk line to obtain pale yellow oily compound, which was dried at $70^{\circ} \mathrm{C} .(2.872 \mathrm{~g}, 80.6 \%)$. Elemental analysis: (Found: $\mathrm{C}$ 83.63, H 8.77, N 3.42. $\mathrm{C}_{28} \mathrm{H}_{35} \mathrm{NO}$ requires C 83.74, H 8.785, N 3.49. ${ }^{1} \mathrm{H}$ NMR (300 MHz; $\mathrm{CDCl}_{3}$; $\left.298 \mathrm{~K}\right) 1.34(\mathrm{~d}, 6 \mathrm{H}, J=6.87 \mathrm{~Hz}, \operatorname{ArCH}(\mathrm{Me}) \mathrm{N}), 1.47\left(\mathrm{~s}, 9 \mathrm{H}, \mathrm{Ar}^{\mathrm{t} B u}\right) 2.32(\mathrm{~s}, 3 \mathrm{H}, \operatorname{ArMe}), 3.58(\mathrm{q}, 2 \mathrm{H}, J=$ $6.87 \mathrm{~Hz}, \operatorname{ArCH}(\mathrm{Me}) \mathrm{N}), 4.09(\mathrm{br}, 2 \mathrm{H}, \operatorname{ArCH}(\mathrm{Me}) \mathrm{N}), 6.62(\mathrm{~d}, 1 \mathrm{H}, J=7.92 \mathrm{~Hz}, \operatorname{ArH}), 6.90(\mathrm{~d}, 1 \mathrm{H}, J=7.92 \mathrm{~Hz}$, $\mathrm{ArH}), 7.26$ - 7.38 (br, $10 \mathrm{H}, \mathrm{ArH}), 11.05(\mathrm{~s}, 1 \mathrm{H}, \mathrm{ArOH}) .{ }^{13} \mathrm{C}\{\mathrm{H}\}$ NMR $\left(75 \mathrm{MHz} ; \mathrm{CDCl}_{3} ; 298 \mathrm{~K}\right) 16.7\left(\mathrm{ArCMe}_{3}\right)$, $24.9\left(\mathrm{ArCMe}_{3}\right), 31.9(\mathrm{ArCH}(\mathrm{Me}) \mathrm{N}), 34.2(\mathrm{ArCH}(\mathrm{Me}) \mathrm{N}), 55.4\left(\mathrm{ArCH}_{2} \mathrm{~N}\right), 114.9,123.8,124.1,127.1,127.3$, 128.3, 128.8, 143.1, 145.2, $152.2($ all $\mathrm{ArC}$ ).

\subsubsection{HL ${ }^{c}$}

4-Tert-butyl-2-methylphenol (1.510 g, $8.87 \mathrm{mmol}), 37 \mathrm{wt} \%$ formaldehyde (0.266 g, $8.87 \mathrm{mmol})$, and (+)-bis- 
[(R)-1-phenylethyl]amine (2.000 g, $8.87 \mathrm{mmol})$ were dissolved in ethanol $(13 \mathrm{~mL})$. The resulting solution was heated at reflux for $18 \mathrm{~h}$ and then cooled to room temperature. Solvent and water were removed using high vacuum Schlenk line to obtain pale yellow oily compound, which was dried at $70^{\circ} \mathrm{C} .(2.133 \mathrm{~g}, 59.9 \%)$. Elemental analysis: (Found: C 83.47, H 8.95, N 3.68. $\mathrm{C}_{28} \mathrm{H}_{35} \mathrm{NO}$ requires C 83.74, H 8.785, N 3.49. ${ }^{1} \mathrm{H}$ NMR (300 MHz; $\mathrm{CDCl}_{3}$; $\left.298 \mathrm{~K}\right)$ 1.41-1.46 (s, 15H, $\left.\operatorname{Ar}^{\mathrm{t}} \mathrm{Bu}, \operatorname{ArCH}(M e) \mathrm{N}\right), 2.44(\mathrm{~s}, 3 \mathrm{H}, \operatorname{ArMe}), 3.67$ (q, 2H, $J=6.87 \mathrm{~Hz}$, $\operatorname{ArCH}(\mathrm{Me}) \mathrm{N}), 4.36$ (br, 2H, $\operatorname{ArCH}(\mathrm{Me}) \mathrm{N}), 6.84(\mathrm{~d}, 1 \mathrm{H}, J=8.25 \mathrm{~Hz}, \operatorname{ArH}), 7.20$ (d, $1 \mathrm{H}, J=8.25 \mathrm{~Hz}, \mathrm{ArH}), 7.33$ 0.46 (br, 10H, ArH), 10.52 (s, $1 \mathrm{H}, \mathrm{ArOH}) .{ }^{13} \mathrm{C}\{\mathrm{H}\}$ NMR (75 MHz; CDCl $\left.; 298 \mathrm{~K}\right) 16.7\left(\mathrm{ArCMe}_{3}\right), 24.9$ $\left(\mathrm{ArCMe}_{3}\right), 31.9(\mathrm{ArCH}(\mathrm{Me}) \mathrm{N}), 34.2(\mathrm{ArCH}(\mathrm{Me}) \mathrm{N}), 55.4\left(\mathrm{ArCH}_{2} \mathrm{~N}\right), 114.9,123.8,124.1,127.1,127.3,128.3$, 128.8, 143.1, 145.2, 152.2 (all $\operatorname{ArC}$ ).

\subsubsection{HL}

2,4-Di-tert-butylphenol (3.522 g, $17.069 \mathrm{mmol}$ ), $37 \mathrm{wt} \%$ formaldehyde (0.512 g, $17.069 \mathrm{mmol}$ ), and N-methylbenzylamine (2.068 g, $17.069 \mathrm{mmol})$ were dissolved in ethanol $(30 \mathrm{~mL})$. The resulting solution was heated at reflux for $18 \mathrm{~h}$ and then cooled to room temperature. Crystallization from the saturated ethanol solution at room temperature yielded white solid, which was dried under high vacuum at $70^{\circ} \mathrm{C}(4.510 \mathrm{~g}, 77.8 \%)$. Mp: $132.6^{\circ} \mathrm{C}$ $132.9^{\circ} \mathrm{C}$. Elemental analysis: (Found: $\mathrm{C} 80.97, \mathrm{H}$ 9.67, N 4.15. $\mathrm{C}_{23} \mathrm{H}_{33} \mathrm{NO}$ requires C 81.37, H 9.80, N 4.13\%). ${ }^{1} \mathrm{H}$ NMR (500 MHz; CDCl 3 ; $298 \mathrm{~K}$ ) 1.43 (s, 9H, $\mathrm{Ar}^{\mathrm{t}} \mathrm{Bu}$ ), 1.64 (s, 9H, $\mathrm{Ar}^{\mathrm{t}} \mathrm{Bu}$ ), 2.29 (s, 3H, $\mathrm{ArCH}_{2} \mathrm{NMe}$ ), 3.61 (br, 2H, $\mathrm{ArCH}_{2} \mathrm{NMe}$ ), 3.78 (br, 2H, $\mathrm{ArCH}_{2} \mathrm{NMe}$ ), 6.92 (s, $1 \mathrm{H}, \mathrm{ArH}$ ), 7.29-7.40 (br, 6H, ArH), 11.17 (br, $1 \mathrm{H}$, $\mathrm{ArOH}){ }^{13} \mathrm{C}\{\mathrm{H}\}$ NMR $\left(125 \mathrm{MHz} ; \mathrm{CDCl}_{3} ; 298 \mathrm{~K}\right) 29.7\left(\mathrm{ArCMe}_{3}\right), 31.4\left(\mathrm{ArCMe}_{3}\right), 34.2\left(\mathrm{ArCMe}_{3}\right), 34.4$ $\left(\mathrm{ArCMe}_{3}\right), 41.2\left(\mathrm{ArCH}_{2} \mathrm{NMe}\right), 60.9\left(\mathrm{ArCH}_{2} \mathrm{NMe}\right), 62.1\left(\mathrm{ArCH}_{2} \mathrm{NMe}\right), 121.4,122.9,123.4,1275,128.5,129.5$, 135.7, 137.3, 140.6, 154.3, 176.6 (all ArC).

\subsubsection{HL}

2,4-Di-tert-pentylphenol (2.000 g, $8.535 \mathrm{mmol}), 37 \mathrm{wt} \%$ formaldehyde $(0.256 \mathrm{~g}, 8.535 \mathrm{mmol})$, and N-methylbenzylamine $(1.034 \mathrm{~g}, 8.535 \mathrm{mmol})$ were dissolved in ethanol $(30 \mathrm{~mL})$. The resulting solution was heated at reflux for $18 \mathrm{~h}$ and then cooled to room temperature. Crystallization from the saturated ethanol solution at $-10^{\circ} \mathrm{C}$ (freezer) yielded white solid, which was dried under high vacuum at $50^{\circ} \mathrm{C}(2.490 \mathrm{~g}, 79.4 \%)$. Mp: $73.9^{\circ} \mathrm{C}-$ 74.1 ${ }^{\circ} \mathrm{C}$. Elemental analysis: (Found: $\mathrm{C} 81.61, \mathrm{H} 9.99, \mathrm{~N} 3.90 . \mathrm{C}_{25} \mathrm{H}_{37} \mathrm{NO}$ requires $\mathrm{C} 81.69, \mathrm{H} 10.15, \mathrm{~N} 3.81 \%$ ). ${ }^{1} \mathrm{H}$ NMR (300 MHz; $\mathrm{CDCl}_{3}$; $\left.298 \mathrm{~K}\right){ }^{1} \mathrm{H}$ NMR (500 MHz; $\left.\mathrm{CDCl}_{3} ; 298 \mathrm{~K}\right) 0.74$ (t, 3H x 2, J = $7.50 \mathrm{~Hz}$, $\mathrm{ArCMe}_{2} \mathrm{CH}_{2} \mathrm{Me}$ ), 1.31 (s, 6H, $\operatorname{ArCMe}_{2} \mathrm{CH}_{2} \mathrm{Me}$ ), 1.47 (s, 6H, $\operatorname{ArCMe}_{2} \mathrm{CH}_{2} \mathrm{Me}$ ), 1.65 (q, 2H, $J=7.50 \mathrm{~Hz}$, $\mathrm{ArCMe}_{2} \mathrm{CH}_{2} \mathrm{Me}$ ), 2.03 (q, $2 \mathrm{H}, J=7.50 \mathrm{~Hz}, \mathrm{ArCMe}_{2} \mathrm{CH}_{2} \mathrm{Me}$ ), 2.27 (s, 3H, $\mathrm{ArCH}_{2} \mathrm{NMe}$ ), 3.57 (br, $2 \mathrm{H}$, $\mathrm{ArCH}_{2} \mathrm{NMe}$ ), 3.79 (br, 2H, $\mathrm{ArCH}_{2} \mathrm{NMe}$ ), 6.86 (s, $\left.1 \mathrm{H}, \mathrm{ArH}\right), 7.16$ (s, 1H, $\left.\mathrm{ArH}\right), 7.31$ - 7.41 (br, 5H, $\left.\mathrm{ArH}\right), 11.04$ (br, $1 \mathrm{H}, \mathrm{ArOH}) .{ }^{13} \mathrm{C}\{\mathrm{H}\}$ NMR $\left(125 \mathrm{MHz} ; \mathrm{CDCl}_{3} ; 298 \mathrm{~K}\right) 9.2\left(\mathrm{ArCMe}_{2} \mathrm{CH}_{2} \mathrm{Me}\right), 9.6\left(\mathrm{ArCMe}_{2} \mathrm{CH}_{2} \mathrm{Me}\right), 27.7$ ( $\left.\mathrm{ArCMe}_{2} \mathrm{CH}_{2} \mathrm{Me}\right)$, $28.6\left(\mathrm{ArCMe}_{2} \mathrm{CH}_{2} \mathrm{Me}\right)$, $33.0\left(\mathrm{ArCMe}_{2} \mathrm{CH}_{2} \mathrm{Me}\right), 37.3\left(\mathrm{ArCMe}_{2} \mathrm{CH}_{2} \mathrm{Me}\right), 38.5\left(\mathrm{ArCMe}_{2} \mathrm{CH}_{2} \mathrm{Me}\right)$, $41.1\left(\mathrm{ArCH}_{2} \mathrm{NMe}\right), 60.8\left(\mathrm{ArCH}_{2} \mathrm{NMe}\right), 62.1\left(\mathrm{ArCH}_{2} \mathrm{NMe}\right), 121.1,124.1,125.1,127.5,128.4,129.5,133.9,137.6$, 138.7154 .0 (all $\mathrm{ArC}$ ).

\subsection{6. $\mathrm{HL}^{\mathrm{f}}$}

2,4-Di-methylphenol (10.421 g, $85.3 \mathrm{mmol}$ ), $37 \mathrm{wt} \%$ formaldehyde (2.56 g, $85.3 \mathrm{mmol}$ ), and N-methylbenzylamine (10.342 g, $85.3 \mathrm{mmol}$ ) were dissolved in methanol $(20 \mathrm{~mL})$. The resulting solution was heated at reflux for $18 \mathrm{~h}$ and then cooled to room temperature. Solvent and water were removed using high vacuum Schlenk line to obtain pale yellow oily solid. Recrystallization from ethanol at $-10^{\circ} \mathrm{C}$ (freezer) yielded off white solid, which was dried under high vacuum at room temperature. (20.45 g, 96.5\%). Mp: $33.5^{\circ} \mathrm{C}-33.6^{\circ} \mathrm{C}$. Elemental analysis: (Found: C 80.01, H 8.13, N 5.50. $\mathrm{C}_{17} \mathrm{H}_{21} \mathrm{NO}$ requires C 79.960, H 8.289, N 5.485. ${ }^{1} \mathrm{H}$ NMR (300 MHz; $\mathrm{CDCl}_{3}$; $298 \mathrm{~K}$ ) 2.22 (s, 3H, ArMe), 2.23 (s, 3H, ArMe), 2.24 (s, 3H, $\mathrm{ArCH}_{2} \mathrm{NMe}$ ), 3.61 (s, 2H, $\mathrm{ArCH}_{2} \mathrm{NMe}$ ), 3.71 (s, $2 \mathrm{H}$, $\mathrm{ArCH}_{2} \mathrm{NMe}$ ), 6.67 (s, $\left.1 \mathrm{H}, \mathrm{ArH}\right), 6.88$ (s, $\left.1 \mathrm{H}, \mathrm{ArH}\right), 7.29-7.37$ (br, 5H, $\left.\mathrm{ArH}\right), 10.97$ (br, $\left.1 \mathrm{H}, \mathrm{ArOH}\right) .{ }^{13} \mathrm{C}\{\mathrm{H}\}$ NMR (125 MHz; CDCl $; 298 \mathrm{~K}) 15.8$ (ArMe), $20.6(\mathrm{ArMe}), 41.2\left(\mathrm{ArCH}_{2} \mathrm{NMe}\right), 61.1\left(\mathrm{ArCH}_{2} \mathrm{NMe}\right), 61.7$ ( $\left.\mathrm{ArCH}_{2} \mathrm{NMe}\right), 120.9,124.8,126.8,127.7,128.9,129.5,130.7,137.0,153.6$ (all $\mathrm{ArC}$ ).

\section{Result and Discussion}

The chiral ligands $\mathrm{HL}^{\mathrm{a}}$, $\mathrm{HL}^{\mathrm{b}}$ and $\mathrm{HL}^{\mathrm{c}}$ (Figure 1) were synthesized via Mannich condensation reactions using inexpensive substituted phenols, formaldehyde and (+)-bis-[(R)-1-phenylethyl] amine in refluxing ethanol (Scheme 1). 


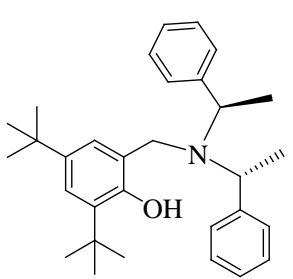

HL $^{\mathrm{a}}$<smiles>CN(Cc1ccccc1)Cc1cc(C(C)(C)C)cc(C(C)(C)C)c1O</smiles>

HL $^{\mathrm{d}}$<smiles>Cc1cc(CN(C(C)c2ccccc2)[C@@H](C)c2ccccc2)c(O)c(C(C)(C)C)c1</smiles>

$\mathbf{H L}^{\mathrm{b}}$<smiles>Cc1cc(C(C)(C)C)cc(CN(C(C)c2ccccc2)[C@@H](C)c2ccccc2)c1O</smiles>

HL $^{\mathrm{c}}$

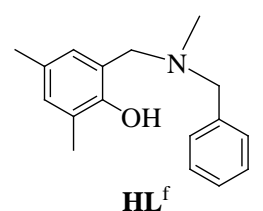

$H^{\mathrm{f}}$
Figure 1. New multidentate ancillary phenolate ligands.<smiles>[R]c1ccc(O)c([R2])c1</smiles><smiles>CC(c1ccccc1)N(N)c1ccccc1</smiles><smiles>CCCCCCCC(C)CC(C)c1ccccc1</smiles>

$\mathrm{HL}^{\mathrm{a}}=\mathrm{R}_{1}=\mathrm{R}_{2}={ }^{\mathrm{t}} \mathrm{Bu}$ $\mathrm{HL}^{\mathrm{b}}=\mathrm{R}_{1}=\mathrm{Me} ; \mathrm{R}_{2}={ }^{\mathrm{t}} \mathrm{Bu}$ $H^{C}{ }^{c}=R_{1}={ }^{\mathrm{t}} \mathrm{Bu} ; \mathrm{R}_{2}=\mathrm{Me}$<smiles>[R]c1cc([R2])c(O)c(CN(C(C)c2ccccc2)[C@@H](C)c2ccccc2)c1</smiles>

HL

Scheme 1. Synthesis of chiral phenolate ligands via Mannich condensation reactions.

These compounds were purified by column chromatography to obtain oily products in moderate to high yields. The corresponding products were then characterized using NMR and elemental analysis to ascertain the structures. The use of different phenolic substituents (methy, butyl and pentyl) will provide a library of compounds suitable for metal catalytic investigations in organic functional group transformations and polymerization reactions.

Meanwhile, the non-chiral ligands $\mathrm{HL}^{\mathrm{d}}, \mathrm{HL}^{\mathrm{e}}$ and $\mathrm{HL}^{\mathrm{f}}$ (Figure 1) were synthesized in a similar manner using substituted phenols, formaldehyde and $\mathrm{N}$-methylbenzylamine in refluxing ethanol. These compounds were purified by recrystallization to obtain white solids in high yields. The solids were also characterized using NMR and elemental analysis. Yields of the non-chiral ligands were generally higher (77\% - 96\%), presumably due to the use of a less bulky N-methylbenzylamine compared to the bulky (+)-bis-[(R)-1-phenylethyl] amine and also due to easier purification methods.

Deprotonation of the ligands and attachment to zinc, tin and palladium metals would offer new research opportunities in asymmetric synthesis and metal catalyzed ring-opening polymerization of lactones. There is great interest in investigating the effect of one stereogenic center in conjunction with phenolic bulky substituent on catalytic selectivity. These ligands are expected to be bidentate with the possibility of having a tridentate coordination via the phenyl pendant arms.

\section{Conclusion}

New chiral monoanionic [ON] ancillary phenolate ligands with varying pendant arms have been synthesized and characterized via nuclear magnetic resonance spectroscopy $\left({ }^{1} \mathrm{H}\right.$ and $\left.{ }^{13} \mathrm{C}\right)$ and elemental analysis. The synthesized ligands are suitable candidates for applications in asymmetric catalysis and ring-opening polymerization of lactones.

\section{Acknowledgements}

The authors are grateful to Duke Energy Advance SC for research funding. 


\section{References}

[1] Walsh, P.J. and Kozlowski, M.C. (2008) Fundamentals in Asymmetric Catalysis. USB, Sausalito. http://dx.doi.org/10.1007/978-3-642-58571-5

[2] Jacobsen, E.N., Pfaltz, A. and Yamamoto, H. (1999) Comprehensive Asymmetric Catalysis. Springer, Heidelberg.

[3] Martin, R., Buchwald, S.L. (2008) Palladium-Catalyzed Suzuki-Miyaura Cross-Coupling Reactions Employing Dialkylbiaryl Phosphine Ligands. Accounts of Chemical Research, 41, 1461-1473. http://dx.doi.org/10.1021/ar800036s

[4] Heitbaum, M., Glorius, F. and Escher, I. (2006) Asymmetric Heterogeneous Catalysis. Angewandte Chemie International Edition, 45, 4732-4762. http://dx.doi.org/10.1002/anie.200504212

[5] Miura, M. (2004) Rational Ligand Design in Constructing Efficient Catalyst Systems for Suzuki-Miyaura Coupling. Angewandte Chemie International Edition, 43, 2201-2203. http://dx.doi.org/10.1002/anie.200301753

[6] Katz, B.A., Cass, R.T., Liu, B., Arze, R. and Collins, N. (1995) Topochemical Catalysis Achieved by Structure-Based Ligand Design. The Journal of Biological Chemistry, 270, 31210-31218.

[7] Falciola, C.A. and Alexakis, A. (2008) Copper-Catalyzed Asymmetric Allylic Alkylation. European Journal of Organic Chemistry, 3765-3780. http://dx.doi.org/10.1002/ejoc.200800025

[8] Hoveyda, A.H., Hird, A.W. and Kacprzynski, M.A. (2004) Small Peptides as Ligands for Catalytic Asymmetric Alkylations of Olefins. Chemical Communications, 1779-1883. http://dx.doi.org/10.1039/b401123f

[9] Lu, Y., Johnstone, T.C. and Arndtsen, B.A. (2009) Hydrogen-Bonding Asymmetric Metal Catalysis with $\alpha$-Amino Acids: A Simple and Tunable Approach to High Enantioinduction. Journal of the American Chemical Society, 131, 11284-11285. http://dx.doi.org/10.1021/ja904185b

[10] Shi, B.-F., Maugel, N., Zhang, Y.-H. and Yu, J.-Q. (2008) Pd ${ }^{\mathrm{II}}$-Catalyzed Enantioselective Activation of C(sp $\left.{ }^{2}\right)-\mathrm{H}$ and $\mathrm{C}\left(\mathrm{sp}^{3}\right)-\mathrm{H}$ Bonds Using Monoprotected Amino Acids as Chiral Ligands. Angewandte Chemie International Edition, 47, 4882-4886. http://dx.doi.org/10.1002/anie.200801030

[11] Blaser, H.-U., Pugin, B., Spindler, F. and Thommen, M. (2007) From a Chiral Switch to a Ligand Portfolio for Asymmetric Catalysis. Accounts of Chemical Research, 40, 1240-1250. http://dx.doi.org/10.1021/ar7001057

[12] Cesar, V., Bellemin-Laponnaz, S., Wadepohl, H. and Gade, L.H. (2005) Designing the "Search Pathway" in the Development of a New Class of Highly Efficient Stereoselective Hydrosilylation Catalysts. Chemistry-A European Journal, 11, 2862-2873. http://dx.doi.org/10.1002/chem.200500132

[13] Delbridge, E.E., Dugah, D.T., Nelson, C.R., Skelton, B.W. and White, A.H. (2007) Synthesis, Structure and Oxidation of New Ytterbium(II) Bis(phenolate) Compounds and Their Catalytic Activity Towards Epsilon-Caprolactone. Dalton Transactions, No. 1, 143-153.

[14] Binda, P.I. and Delbridge, E.E. (2007) Synthesis and Characterisation of Lanthanide Phenolate Compounds and Their Catalytic Activity towards Ring-Opening Polymerisation of Cyclic Esters. Dalton Transactions, 41, 4685-4692. http://dx.doi.org/10.1039/b710070a

[15] Kerton, F.M., Whitwood, A.C. and Willans, C.E. (2004) A High-Throughput Approach to Lanthanide Complexes and Their Rapid Screening in the Ring Opening Polymerisation of Caprolactone. Dalton Transactions, No. 15, $2237-2244$. http://dx.doi.org/10.1039/b406841f

[16] Binda, P.I., Delbridge, E.E., Abrahamson, H.B. and Skelton, B.W. (2009) Coordination of Substitutionally Inert Phenolate Ligands to Lanthanide(II) and (III) Compounds-Catalysts for Ring-Opening Polymerization of Cyclic Esters. Dalton Transactions, No. 15, 2777-2787. http://dx.doi.org/10.1039/b821770j

[17] Dugah, D.T., Skelton, B.W. and Delbridge, E.E. (2009) Synthesis and Characterization of New Divalent Lanthanide Complexes Supported by Amine Bis(Phenolate) Ligands and Their Applications in the Ring Opening Polymerization of Cyclic Esters Dalton Transactions, 1436-1445. http://dx.doi.org/10.1039/b816916k

[18] Xu, X., Ma, M., Yao, Y., Zhang, Y. and Shen, Q. (2005) Synthesis, Characterisation of Carbon-Bridged (Diphenolato) Lanthanide Complexes and Their Catalytic Activity for Diels-Alder Reactions. European Journal of Inorganic Chemistry, 2005, 676-684. http://dx.doi.org/10.1002/ejic.200400519

[19] Lendlein, A., Schmidt, A.M., Schroeter, M. and Langer, R. (2005) Shape-Memory Polymer Networks from Oligo ( $\epsilon$-Caprolactone) Dimethacrylates. Journal of Polymer Science Part A: Polymer Chemistry, 43, 1369-1381. http://dx.doi.org/10.1002/pola.20598

[20] Amgoune, A., Thomas, C.M., Roisnel, T. and Carpentier, J.F. (2005) Ring-Opening Polymerization of Lactide with Group 3 Metal Complexes Supported by Dianionic Alkoxy-Amino-Bisphenolate Ligands: Combining High Activity, Productivity and Selectivity. Chemistry —A European Journal, 12, 169-179. http://dx.doi.org/10.1002/chem.200500856

[21] Guo, H., Zhou, H., Yao, Y., Zhang, Y. and Shen, Q. (2007) Synthesis and Structural Characterization of Novel Mixed- 
Valent Samarium and Divalent Ytterbium and Europium Complexes Supported by Amine Bis(phenolate) Ligand. Dalton Transactions, No. 32, 3555-3561. http://dx.doi.org/10.1039/b705353c

[22] Zats, G.M., Arora, H., Lavi, R., Yufit, D. and Benisvy, L. (2011) Phenolate and Phenoxyl Radical Complexes of Cu(II) and Co(III), Bearing a New Redox Active N,O-Phenol-Pyrazole Ligand. Dalton Transactions, 40, 10889-10896. http://dx.doi.org/10.1039/c1dt10615e

[23] Radano, C.P., Baker, G.L. and Smith, M.R. (2000) Stereoselective Polymerization of a Racemic Monomer with a Racemic Catalyst: Direct Preparation of the Polylactic Acid Stereocomplex from Racemic Lactide. Journal of the American Chemical Society, 122, 1552-1553. http://dx.doi.org/10.1021/ja9930519

[24] Darensbourg, D.J., Choi, W., Karroonnirum, O. and Bhuvanesh, N. (2008) Ring-Opening Polymerization of Cyclic Monomers by Complexes Derived from Biocompatible Metals. Production of Poly(lactide), Poly(trimethylene carbonate) and Their Copolymers. Macromolecules, 41, 3493-3502. http://dx.doi.org/10.1021/ma800078t

[25] Ovitt, T.M. and Coates, G.W. (2002) Stereochemistry of Lactide Polymerization with Chiral Catalysts: New Opportunities for Stereocontrol Using Polymer Exchange Mechanisms. Journal of the American Chemical Society, 124, 13161326. http://dx.doi.org/10.1021/ja012052+

[26] Kasperczyk, J.E. (1995) Microstructure Analysis of Poly(lactic acid) Obtained by Lithium Tert-Butoxide as Initiator. Macromolecules, 28, 3937-3939. http://dx.doi.org/10.1021/ma00115a028

[27] Radano, C.P., Baker, G.L. and Smith III, M.R. (2000) Stereoselective Polymerization of a Racemic Monomer with a Racemic Catalyst: Direct Preparation of the Polylactic Acid Stereocomplex from Racemic Lactide. Journal of the American Chemical Society, 12, 1552-1553. http://dx.doi.org/10.1021/ja9930519

[28] Thakur, K.A.M., Kean, R.T., Zell, M.T., Padden, B.E. and Munson, E.J. (1998) An Alternative Interpretation of the HETCOR NMR Spectra of Poly(lactide). Chemical Communications, No. 17, 1913-1914. http://dx.doi.org/10.1039/a708911b

[29] Thakur, K.A.M., Kean, R.T., Hall, E.S., Kolstad, J.J. and Lindgren, T.A. (1997) High-Resolution ${ }^{13} \mathrm{C}$ and ${ }^{1} \mathrm{H}$ Solution NMR Study of Poly(lactide). Macromolecules, 30, 2422-2428. http://dx.doi.org/10.1021/ma9615967

[30] Zell, M.T., Padden, B.E., Paterick, A.J., Thakur, K.A.M., Kean, R.T., Hillmyer, M.A. and Munson, E.J. (2002) Unambiguous Determination of the ${ }^{13} \mathrm{C}$ and ${ }^{1} \mathrm{H}$ NMR Stereosequence Assignments of Polylactide Using High-Resolution Solution NMR Spectroscopy. Macromolecules, 35, 7700-7707. http://dx.doi.org/10.1021/ma0204148

[31] Ovitt, T.M. and Coates, G.W. (2000) Stereoselective Ring-Opening Polymerization of Rac-Lactide with a Single-Site, Racemic Aluminum Alkoxide Catalyst: Synthesis of Stereoblock Poly(lactic acid), Polymer Chemistry, 38, 4686-4692. http://dx.doi.org/10.1002/1099-0518(200012)38:1+<4686::AID-POLA80>3.0.CO;2-0

[32] Cheng, M., Attygalle, A.B., Lobkovsky, E.B. and Coates, G.W. (1999) Single-Site Catalysts for Ring-Opening Polymerization: Synthesis of Heterotactic Poly(lactic acid) from Rac-Lactide. Journal of the American Chemical Society, 121, 11583-11584. http://dx.doi.org/10.1021/ja9926780

[33] Ovitt, T.M and Coates, G.W. (1999) Stereoselective Ring-Opening Polymerization of meso-Lactide: Synthesis of Syndiotactic Poly(lactic acid). Journal of the American Chemical Society, 121, 4072-4073. http://dx.doi.org/10.1021/ja990088k

[34] Chisholm, M.H., Iyer, S.S., McCollum, D.G., Pagel, M. and Werner-Zwanziger, U. (1999) Microstructure of Poly(lactide). Phase-Sensitive HETCOR Spectra of Poly(meso-lactide), Poly(rac-lactide) and Atactic Poly(lactide). Macromolecules, 32, 963-973. http://dx.doi.org/10.1021/ma9806864

[35] Chisholm, M.H., Gallucci, J.C. and Phomphrai, K. (2005) Comparative Study of the Coordination Chemistry and Lactide Polymerization of Alkoxide and Amide Complexes of Zinc and Magnesium with a $\beta$-Diiminato Ligand Bearing Ether Substituents. Inorganic Chemistry, 44, 8004-8010. http://dx.doi.org/10.1021/ic048363d

[36] Zhong, Z., Dijkstra, P.J. and Feijen, J. (2003) Controlled and Stereoselective Polymerization of Lactide: Kinetics, Selectivity, and Microstructures. Journal of the American Chemical Society, 125, 11291-11298. http://dx.doi.org/10.1021/ja0347585

[37] Cai, C.-X., Amgoune, A., Lehmann, C.W. and Carpentier, J.-F. (2004) Stereoselective Ring-Opening Polymerization of Racemic Lactide Using Alkoxy-Amino-Bis(phenolate) Group 3 Metal Complexes. Chemical Communications, No. 3, 330-331. http://dx.doi.org/10.1039/b314030j

[38] Coates, G.W. (2000) Precise Control of Polyolefin Stereochemistry Using Single-Site Metal Catalysts. Chemical Reviews, 100, 1223-1252. http://dx.doi.org/10.1021/cr990286u

[39] Nimitsiriwat, N., Marshall, E.L., Gibson, V.C., Elsegood, M.R.J. and Dale, S.H. (2004) Unprecedented Reversible Migration of Amide to Schiff Base Ligands Attached to Tin: Latent Single-Site Initiators for Lactide Polymerization. Journal of the American Chemical Society, 126, 13598-13599. http://dx.doi.org/10.1021/ja0470315

[40] Chisholm, M.H., Eilerts, N.W., Huffman, J.C., Iyer, S.S., Pacold, M. and Phomphrai, K. (2000) Molecular Design of Single-Site Metal Alkoxide Catalyst Precursors for Ring-Opening Polymerization Reactions Leading to Polyoxyge- 
nates. 1. Polylactide Formation by Achiral and Chiral Magnesium and Zinc Alkoxides, $\left(\eta^{3}-\mathrm{L}\right) \mathrm{MOR}$, Where L=Trispyrazolyl- and Trisindazolylborate Ligands. Journal of the American Chemical Society, 122, 11845-11854.

http://dx.doi.org/10.1021/ja002160g

[41] Cai, C.X., Toupet, L., Lehmann, C.W. and Carpentier, J.-F. (2003) Synthesis, Structure and Reactivity of New Yttrium Bis(dimethylsilyl) Amido and Bis(trimethylsilyl) Methyl Complexes of a Tetradentate Bis(phenoxide) Ligand. Journal of Organometallic Chemistry, 683, 131-136. http://dx.doi.org/10.1016/S0022-328X(03)00513-8

[42] Chamberlain, B.M., Cheng, M., Moore, D.R., Ovitt, T.M., Lobkovsky, E.B. and Coates, G.W. (2001) Polymerization of Lactide with Zinc and Magnesium $\beta$-Diiminate Complexes: Stereocontrol and Mechanism. Journal of the American Chemical Society, 123, 3229-3238. http://dx.doi.org/10.1021/ja003851f

[43] Kowalski, A., Duda, A. and Penczek, S. (1998) Polymerization of L,L-Lactide Initiated by Aluminum Isopropoxide Trimer or Tetramer. Macromolecules, 31, 2114-2122. http://dx.doi.org/10.1021/ma971737k

[44] Dove, A.P., Gibson, V.C., Marshall, E.L., White, A.J.P. and Williams, D. (2001) A Well Defined Tin(II) Initiator for the Living Polymerisation of Lactide. Chemical Communications, No. 3, 283-284. http://dx.doi.org/10.1039/b008770j

[45] Grunova, E., Kirillov, E., Roisnel, T. and Carpentier, J.-F. (2008) Group 3 Metal Complexes of Salen-Like FluorousDialkoxy-Diimino Ligands: Synthesis, Structure and Application in Ring-Opening Polymerization of rac-Lactide and rac- $\beta$-Butyrolactone. Organometallics, 27, 5691-5698. http://dx.doi.org/10.1021/om800611c

[46] Binda, P.I., Delbridge, E.E., Dugah, D.T., Skelton, B.W. and White, A.H. (2008) Synthesis and Structural Characterization of Some Potassium Complexes of Some Bis(phenolate) Ligands and Some Novel Heterobimetallic Binuclear Arrays Formed with Trivalent Lanthanoid Ions. Zeitschrift für anorganische und allgemeine Chemie, 634, 325-334. http://dx.doi.org/10.1002/zaac.200700442

[47] Cortright, S.B. and Johnston, J.N. (2002) IAN-Amines: Direct Entry to a Chiral $C_{2}$-Symmetric Zirconium(IV) $\beta$-Diketimine Complex. Angewandte Chemie International Edition, 41, 345-348. http://dx.doi.org/10.1002/1521-3773(20020118)41:2<345::AID-ANIE345>3.0.CO;2-U. 
Scientific Research Publishing (SCIRP) is one of the largest Open Access journal publishers. It is currently publishing more than 200 open access, online, peer-reviewed journals covering a wide range of academic disciplines. SCIRP serves the worldwide academic communities and contributes to the progress and application of science with its publication.

Other selected journals from SCIRP are listed as below. Submit your manuscript to us via either submit@scirp.org or Online Submission Portal.
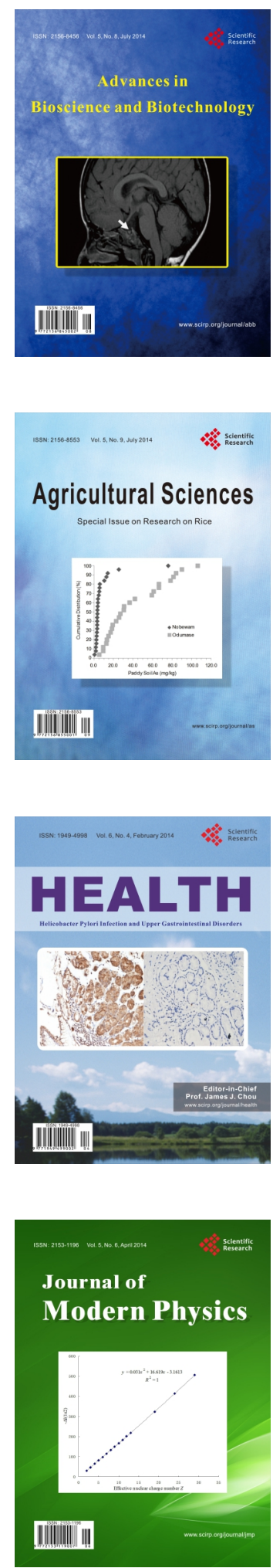
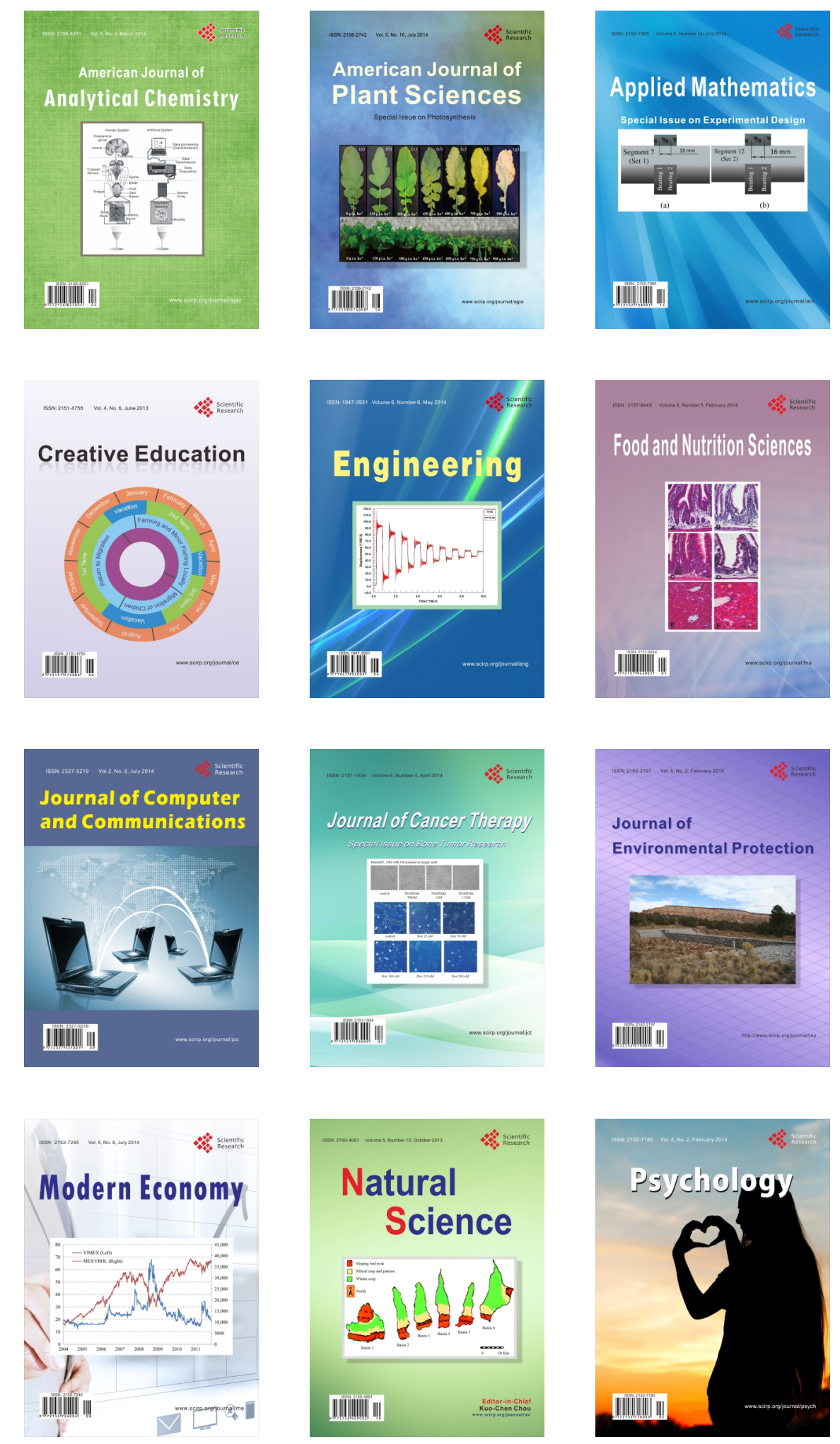